\title{
Movement Identification and Realization about Planar Linkage Mechanisms
}

\author{
Jie $\mathrm{Wu}^{1, \mathrm{a}}$, Zhaomeng Yang ${ }^{1, \mathrm{~b}}$ and Dongzheng $\mathrm{Wang}^{2, \mathrm{c}}$ \\ ${ }^{1}$ School of Mechanical Engineering, Shenyang University, Liaoning 110044, China \\ ${ }^{2}$ Faculty of Electronic information and Electrical Engineering, Dalian University of Technology, \\ Liaoning 116024, China \\ awujied85@163.com, b1852609778@qq.com, c418731866@qq.com
}

Keywords: Planar linkage mechanisms, Movement identification, Coupler-curve.

\begin{abstract}
In this paper, based on the planar four-bar linkage mechanisms as the research object as well as to carry out the kinematics analysis, a kinematics calculation mode of the planar four-bar linkage mechanism was established by the OOP method of Visual Basic. Based on the kinematics calculation mode, a movement identification system of planar four-bar linkage mechanisms was developed. This system cannot only realize the movement identification of random type of four-bar linkage mechanisms, but also can achieve the couple curve of the kinematics simulation of the planar four-bar linkage mechanisms. This provided a practical method of kinematics analysis of the planar four-bar linkage mechanisms.
\end{abstract}

\section{Introduction}

The planar linkage mechanisms are widely used in the machinery. The planar four-bar mechanism is the simplest planar linkage mechanisms. There are four links and four revolute pairs, Four-bar mechanisms are extremely versatile and useful devices. Many complex motion control problems can be solved with four-bar mechanisms.

The motion is known, when the motion law of the follower of the original, according to the given motion; realization of trajectory is known, is the requirement of organization member moving in a plane on a point along a given trajectory. Calculation and Simulation of traditional design, the simulation calculation is complex, heavy workload, limited the precise application of four bar mechanism, the powerful function of the development of computer technology and related software carrier, greatly expanding the hinge four bar mechanism of traditional precise scope of application. The movement of the mechanism synthesis must be deduced mechanism at different positions in active thing, follower position, velocity, acceleration and member of plane motion of a point on the trajectory, so that with the help of computer simulation.

\section{Kinematic Analysis of Linkage Mechanisms}

\section{Composition of Linkage Mechanisms}

The planar four-bar mechanism is the simplest planar linkage mechanisms. If all lower pairs in a four-bar mechanism are revolute pairs, as shown in Fig1, The mechanism is called a revolute four-bar mechanism, which is the basic form of the four-bar mechanism. According to the types of the two side links, the types of revolute four-bar mechanisms can be divided into: crank-rocker mechanism, Double-crank mechanism and Double- rocker mechanism. The forms of motion are different.

There are many kinds of analytical methods for kinematic analysis of four-bar mechanism. the kinematic analysis of linkage mechanism seems to be a hard task at first sight However, it becomes easier if the Assur-group method is used, Most link age mechanisms are built up by adding one or more commonly used Assur group to the basic mechanism. 


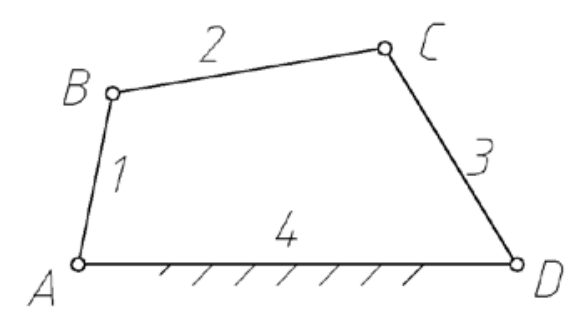

1-crank 2-coupler 3-rocker 4-fram

Fig1 . Composition of Linkage Mechanisms

\section{The Mathematical Model of Linkage Mechanisms}

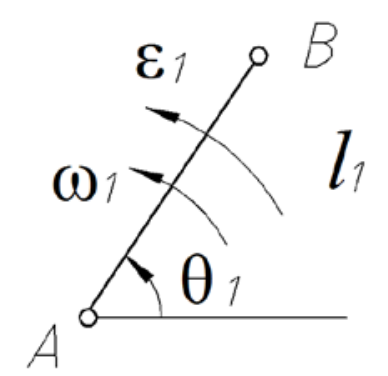

Fig2 . The Schematic Diagram of the Link

Suppose that the $\mathrm{x}$ and $\mathrm{y}$ components of position velocity and acceleration of a point A (i.e. $x_{\mathrm{A}}, y$ ${ }_{\mathrm{A}}, v_{\mathrm{Ax}}, v_{\mathrm{Ay}}, a_{\mathrm{Ax}}, a_{\mathrm{Ay}}$,),the angular position, the angular velocity and the angular acceleration of link $\mathrm{AB}$ (i.e. $\omega_{1}, \varepsilon_{1}, \theta_{1}$ ), and the length $\left(l_{1}\right)$ of the link $\mathrm{AB}$ are known ,as shown in Fig 2, the $\mathrm{x}$ and $\mathrm{y}$ components of position,velocity and acceleration of a point $\mathrm{B}$ (i.e. $\mathrm{B}, y_{\mathrm{B}}, v_{\mathrm{Bx}}, v_{\mathrm{By}}, a_{\mathrm{Bx}}, a_{\mathrm{By}}$,) $c a n$ be calculated as follows.

In a Cartesian co-ordinate system:

$x_{\mathrm{B}}=x_{\mathrm{A}}+l_{1} \cos \theta_{1}$

$y_{\mathrm{B}}=y_{\mathrm{A}}+l_{1} \sin \theta_{1}$

The formulae for analyzing the velocity of the point $\mathrm{B}$ analysis can be derived.

$v_{\mathrm{Bx}}=v_{\mathrm{Ax}}-l_{1}\left(\sin \theta_{1}\right) \omega_{1}$

$v_{\mathrm{By}}=v_{\mathrm{Ay}}+l_{1}\left(\cos \theta_{1}\right) \omega_{1}$

The formulae for acceleration of a point $\mathrm{B}$ analysis can be derived.

$$
\begin{aligned}
& a_{\mathrm{Bx}}=a_{\mathrm{Ax}}-l_{1}\left(\sin \theta_{1}\right) \varepsilon_{1}-l_{1}\left(\cos \theta_{1}\right) \omega_{1}{ }^{2} \\
& a_{\mathrm{By}}=a_{\mathrm{Ay}}+l_{1}\left(\cos \theta_{1}\right) \varepsilon_{1}-l_{1}\left(\sin \theta_{1}\right) \omega_{1}{ }^{2}
\end{aligned}
$$

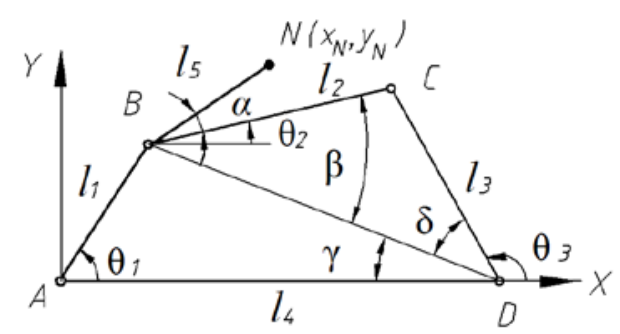

Fig3 . Schematic Diagram of t Linkage Mechanisms

These six formulae can be programmed in a subroutine. In Visual Basic,any subroutine must begin with statement. In linkage mechanisms ABCD as shown in Fig3, thecrank AB is aninput link and rocker CD is an output link .

The Kinematic parameters of the two out point $A$ and $C$ and the length of the two links $A B$ and $B C$ are known. The angular positions, angular velocities, and angular accelerations of the links $A B$ and $\mathrm{BC}$ can be calculated as follows. 
When x A, y A, x C, y C, 11 and 12 are determined, there are two assembly modes for this group,as shown in Fig3.

On the link BC,

$\mathrm{x} B=\mathrm{x} \mathrm{c}+\mathrm{l} 2 \cos \theta 2$

$\mathrm{yB}=\mathrm{y} \mathrm{c}+12 \sin \theta 2$

On the link $A B$,

$\mathrm{x} \mathrm{B}=\mathrm{x} \mathrm{A}+\mathrm{l} 1 \cos \theta 1$

$\mathrm{y} \mathrm{B}=\mathrm{y} \mathrm{A}+\mathrm{l} 1 \sin \theta 1$

Combining these two sets of equations, one obtains:

$\mathrm{x} \mathrm{C}+\mathrm{l} 2 \cos \theta 2=\mathrm{xA}+\mathrm{l} 1 \cos \theta 1$

$\mathrm{y} \mathrm{c}+12 \sin \theta 2=\mathrm{y} \mathrm{A}+11 \sin \theta 1$

There are two unknowns, $\theta 1$ and $\theta 2$, in this set of equation. Since there are two assembly modes for this group, there will be two sets of solutions.

Velocity analysis can be progressed only after the position analysis is finished. The angular velocities, $\omega 1$ and $\omega 2$, of the links $\mathrm{AB}$ and $\mathrm{BC}$ can be found by differentiating Eqs( 4 ).

$\mathrm{v} C \mathrm{Cx}-\mathrm{l} 2(\sin \theta 2) \omega 2=\mathrm{v} A x-\mathrm{l} 1(\sin \theta 1) \omega 1$

$\mathrm{v} \mathrm{Cy}+\mathrm{l} 2(\cos 2) \omega 2=\mathrm{v} A y+\mathrm{l} 1(\cos \theta 1) \omega 1$

The copler point $\mathrm{N}$ (Fig.3)

$x_{N}=l_{1} \cos \left(\theta_{1}\right)+l_{5} \cos \left(\theta_{2}+\alpha\right)$

$y_{N}=l_{1} \sin \left(\theta_{1}\right)+l_{5} \sin \left(\theta_{2}+\alpha\right)$

The angular velocity and the angular acceleration of link BC (i.e. $\omega 2, \varepsilon 2)$

$\omega_{2}=-\omega_{1} \frac{l_{1} \sin \left(\theta_{1}-\theta_{3}\right)}{l_{3} \sin \left(\theta_{2}-\theta_{3}\right)}$

$\varepsilon_{2}=\frac{l_{3} \omega_{3}^{2}-l_{1} \omega_{1}^{2} \cos \left(\theta_{1}-\theta_{3}\right)-l_{2} \omega_{2}^{2} \cos \left(\theta_{2}-\theta_{3}\right)}{l_{2} \sin \left(\theta_{2}-\theta_{3}\right)}$

The angular velocity and the angular acceleration of link CD (i.e. $\omega 3, \varepsilon 3)$

$$
\begin{aligned}
& \omega_{3}=\omega_{1} \frac{l_{1} \sin \left(\theta_{1}-\theta_{2}\right)}{l_{2} \sin \left(\theta_{3}-\theta_{2}\right)} \\
& \varepsilon_{3}=\frac{l_{2} \omega_{2}^{2}+l_{1} \omega_{1}^{2} \cos \left(\theta_{1}-\theta_{2}\right)-l_{3} \omega_{3}^{2} \cos \left(\theta_{3}-\theta_{2}\right)}{l_{3} \sin \left(\theta_{3}-\theta_{2}\right)}
\end{aligned}
$$

The coupler curve coordinates are derived, angular velocity and angular acceleration of the connecting rod and rocker, the calculation is complex and has to use a computer, and the corresponding program.

\section{Path generation of the mechanism}

For any four bar linkage mechanism, when the driver to meet the conditions of crank rod group, RRR can be installed to a given Flag (counterclockwise for 1, clockwise for -1). The mechanism of crank and rocker mechanism or double crank mechanism.

Procedures for the use of automatic identification method, namely when the driver has an incremental and beyond the range of motion, the original is to change the original direction (Flag= Flag) and inversion, so as to realize the motion simulation in the feasible region of the double rocker mechanism. The motion of the mechanism uses the Enabled property of the Timer control to realize the motion of the mechanism and suspension. The program flow diagram as shown in Fig. 4. 


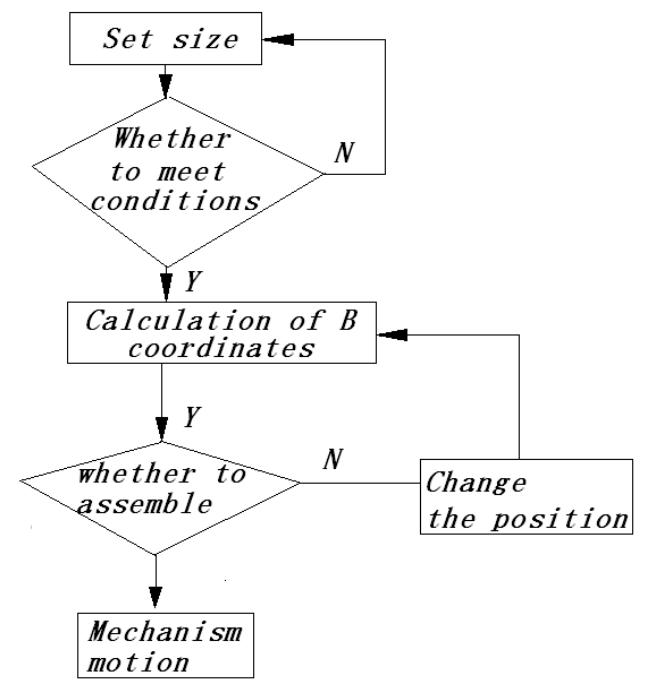

Fig4 . The program flow diagram

With an array of $x_{N}, y_{N}$ record from the beginning of the click of a mouse, the coordinate mechanism in the operation process of the $\mathrm{N}$ point position. The connection of these coordinates, to obtain link curve. As shown in figure 5 . When the connecting rod curve satisfy a requirement, can record the $\mathrm{M}$ size and position of the connecting rod mechanism. This method can be extended to any linkage coupler curve drawing.

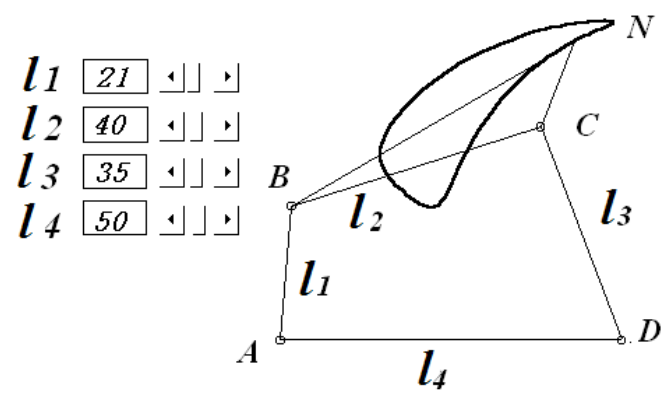

Fig5 coupler curve

\section{Conclusion}

The author used the method of successful preparation of Visual Basic software, to achieve the rendering of motion simulation and the connecting rod curve of arbitrary four bar mechanism. The software has certain reference value for the design of teaching methods and mechanism, at the same time is proposed in this paper can be extended to the synthesis and kinematic demo of complex mechanism.

\section{References}

[1] Wang wen-ming, Zeng Cong-min, Gao Ying-min.et al. simulation System Design of Four Bar Linkage Mechanism. Mechanical and electrical engineering technology.Vol. 1 (2007) No. 1, p. 33-34.

[2] Grosjean J. Kinematics and Dynamism of Mechanisms. McGraw-Hill Inc.1991. p. 67-78.

[3] Ye Zhonghe, Lan Zhaohui, M. R. Smith, et al. Mechanisms And Machine Theory. Higher Education Press, 2014, p.52-66. 